\title{
Comparison of alternative extraction methods for secretome profiling in human hepatocellular carcinoma cells
}

\author{
CAO Jing ${ }^{1}$, SHEN ChengPin ${ }^{1}$, ZHANG Jun ${ }^{1}$, YAO Jun ${ }^{1}$, SHEN HuaLi ${ }^{1}$, LIU YinKun ${ }^{1,2}$, \\ LU HaoJie $^{1} \&$ YANG Peng Yuan ${ }^{1 *}$ \\ ${ }^{1}$ Institutes of Biomedical Sciences and Department of Chemistry, Fudan University, Shanghai 200032, China; \\ ${ }^{2}$ Liver Cancer Institute Zhongshan Hospital, Fudan University, Shanghai 200032, China
}

Received June 11, 2010; accepted August 30, 2010

\begin{abstract}
Secreted proteins are important sources for early detection and diagnosis of disease, and as such have received considerable attention. The extraction of low concentration proteins from large volumes of culture media, which are rich in salts and other compounds that interfere with most proteomics techniques, presents a problem for secretome studies. Ultrafiltration, precipitation, and dialysis are three major extraction methods that can be used to overcome this problem. The present study for the first time, compared the merits and shortcomings of these three methods, without bias. Centrifugal ultrafiltration provided the best extraction efficiency, and precipitation provided the highest number of identifiable proteins. The three methods yielded closely related, but different, information on the secretome; thus, they should be considered complementary or, at least, supplementary methods. Three hundred and sixty unique proteins were identified, including 211 potential secreted proteins. Compared with previous studies, this study also identified 42 new secreted proteins. The present study not only offers a reference for the selection of secretome extraction methods, but also expands the secretome database for the investigation of hepatocellular carcinoma.
\end{abstract}

hepatocellular carcinoma, secretome, protein extraction, ultrafiltration, precipitation, dialysis, LC-MS/MS

Citation: Cao J, Shen C P, Zhang J, et al. Comparison of alternative extraction methods for secretome profiling in human hepatocellular carcinoma cells. Sci China Life Sci, 2011, 54: 34-38, doi: 10.1007/s11427-010-4122-1

The secretome has a pivotal role in many biological processes, including signal transduction, immune defense, cell-cell interaction, cancer cell invasion, and metastasis. Many proteins overexpressed in tumors are secreted proteins [1-3]. For example, osteopontin, a secreted phosphoprotein whose overexpression correlated with the metastatic potential of primary hepatocellular carcinoma (HCC) and with invasiveness of liver tumor-derived cell lines in vitro [4]. Metastasis remains the cause of $90 \%$ of deaths from solid tumors [5]. HCC is no exception, and is one of the most common and aggressive human malignancies [6]; its high mortality rate is mainly the result of intra-hepatic metas-

*Corresponding author (email: pyyang@ fudan.edu.cn) tases.

Thus, the analysis of a cell's secretome could be valuable in diagnosis and prognosis. However, proteins are usually secreted at low concentrations into the culture media, which makes their recovery difficult. Currently, there are three major methods for the purification and concentration of secretory proteins: ultrafiltration [7-9] with molecularweight cut-off membranes, precipitation [10-12] with organic solvents, e.g., trichloroacetic acid (TCA), and dialysis [13-15] with cut-off membranes followed by drying using vacuum centrifugation. Each of these methods has its own disadvantages, and no single one can capture all proteins secreted into the culture media. Accordingly, we used all three methods to investigate the secretome of the human 
HCC cell line, HCCLM3. Accompanied by nano-LC-ESIMS/MS analysis, the three methods were compared in terms of their merits and shortcomings.

\section{Materials and methods}

\subsection{Cell culture}

The HCCLM3 cell line had been established previously [16]. Cells were grown in DMEM culture medium containing $10 \%$ fetal bovine serum until $60 \%-70 \%$ confluence was achieved [17]. The cells were stringently and gently washed, twice with Dulbecco's phosphate buffered saline with calcium and magnesium (DPBS), and once with serum- and phenol red-free DMEM (conditioned medium, CM). The cells were then incubated in $\mathrm{CM}$ at $37^{\circ} \mathrm{C}$. After $24 \mathrm{~h}$, the $\mathrm{CM}$ containing secreted proteins was collected and centrifuged at $1000 \times g$ for $5 \mathrm{~min}\left(4^{\circ} \mathrm{C}\right)$, and then filtered using a $0.45 \mu \mathrm{m}$ filter (Millipore, Bedford, MA, USA) to pellet detached cells and large debris. The supernatant was collected and then centrifuged for $1 \mathrm{~h}$ at $100000 \times g\left(4^{\circ} \mathrm{C}\right)$ to pellet smaller debris and vesicles. TFA $(0.1 \%)$ was immediately added to the final supernatant, which was stored at $-80^{\circ} \mathrm{C}$. The addition of TFA lowered the $\mathrm{pH}(<4)$ of the culture supernatants, thus reducing the activity of many proteases.

\subsection{Preparation of secretory proteins}

The proteins present in the culture supernatants were extracted by various procedures. Twenty milliliters of culture supernatants was used for each method. All the treatments were done at $4^{\circ} \mathrm{C}$. The concentrations of the extracted proteins were measured by the Bradford assay (Bio-Rad Laboratories, Hercules, CA, USA).

\subsubsection{Centrifugal ultrafiltration}

The culture supernatants were concentrated using centriprep centrifugal filter devices with Ultracel YM 3000 Daltons MWCO membrane (Millipore, Bedford, MA, USA) at $3000 \times g$ for $6 \mathrm{~h}$. The resulting concentrate was collected.

\subsubsection{Precipitation}

The culture supernatants were precipitated using a previously described method [18], with minor modifications. Sodium lauroyl sarcosinate (NLS) at $0.5 \%$ final concentration was introduced into the CM solution. After mixing, TCA was added to a final concentration of $12 \%$, and the solution was precipitated on ice for $2 \mathrm{~h}$. The mixed protein-detergent precipitate was collected by centrifugation $\left(10000 \times g, 10 \mathrm{~min}, 4^{\circ} \mathrm{C}\right)$. The supernatant was carefully removed, $2 \mathrm{~mL}$ of tetrahydrofuran (THF) (pre-cooled in ice) was added to the pellet and vortexed until the pellet dislodged from the bottom of the tube and dissolved almost completely. Centrifugation was carried out as described above. The supernatant was removed, and the nearly invisible pellet was washed again with $2 \mathrm{~mL}$ of THF. Finally, the pellet was redissolved in $0.4 \mathrm{~mL}$ extraction solution with the help of a sonicator bath (30 min extraction).

\subsubsection{Dialysis}

The culture supernatants were dialyzed against water (molecular mass cut-off $3500 \mathrm{Da}$; Spectrum, CA, USA) for $48 \mathrm{~h}$ and concentrated using a SpeedVac (CHRIST, Germany).

\subsection{Western blot analysis}

Proteins $(30 \mu \mathrm{g})$ of the culture supernatants and cell extracts were analyzed by Western blotting, as previously described [19]. Proteins were separated on SDS-PAGE, transferred onto PVDF membranes, and probed with antibodies against $\beta$-tubulin, followed by secondary antibody conjugated with horseradish peroxidase. The blot was developed using the Western lightning chemiluminescence reagent (GE Healthcare, USA).

\subsection{In-solution digestion}

The extracted proteins were dissolved and heated at $100^{\circ} \mathrm{C}$ for $10 \mathrm{~min}$. After allowing the sample to cool to room temperature, dithiothreitol (DTT) was introduced into the solution at a final concentration of $10 \mathrm{mmol} \mathrm{L}{ }^{-1}$, and the sample was incubated at $57^{\circ} \mathrm{C}$ for $30 \mathrm{~min}$. To prevent disulfide bond formation, cysteine residues were modified by alkylation with iodoacetamide $\left(20 \mathrm{mmol} \mathrm{L}^{-1}\right.$ final concentration) for $30 \mathrm{~min}$ in the dark, at room temperature. The reaction was quenched by the addition of DTT at half of the molar concentration of the iodoacetamide for $10 \mathrm{~min}$. After iodoacetamide deactivation, the sample solution was diluted 10 folds with $50 \mathrm{mmol} \mathrm{L} \mathrm{NH}_{4} \mathrm{HCO}_{3}$ buffer. Trypsin was added to the sample $(1: 50)$ to digest the proteins overnight (at $37^{\circ} \mathrm{C}$ ). All digested peptide mixtures were dried using vacuum centrifugation and stored at $-20^{\circ} \mathrm{C}$ until analysis by mass spectrometry.

\subsection{LC-MS/MS analysis}

All digested peptide mixtures were resuspended with a solution containing 5\% acetonitrile and $0.1 \%$ formic acid, separated by on-line nanoLC and analyzed by electrospray tandem mass spectrometry. The experiments were performed on an LC-20AD system (Shimadzu, Tokyo, Japan) connected to an LTQ Orbitrap mass spectrometer (Thermo Electron, Bremen, Germany) equipped with a nanoelectrospray ion source (Michrom Bioresources, Auburn, USA). The separation of the peptides took place in a $10-\mathrm{cm}$ column (75- $\mu \mathrm{m}$ inner diameter; New Objective, Woburn, USA) packed with $5 \mu \mathrm{m}$ Biobasic ${ }^{\mathrm{TM}} \mathrm{C} 18$.

The peptide mixtures were injected onto the trap-column 
with a flow of $60 \mu \mathrm{L} \mathrm{min}{ }^{-1}$ and subsequently eluted with a gradient of $5 \%-45 \%$ solvent B $(95 \%$ acetonitrile in $0.1 \%$ formic acid) over $90 \mathrm{~min}$. The peptides were then injected into the mass-spectrometer at a constant column-tip flow rate of $\sim 300 \mathrm{~nL} \mathrm{~min}^{-1}$. Eluted peptides were analyzed by MS and data-dependent MS/MS acquisition, selecting the eight most abundant precursor ions for MS/MS with a dynamic exclusion duration of $1 \mathrm{~min}$. Two biological replicates were performed for each concentration method, and each biological replicate was analyzed four times.

\subsection{Database searching}

The mass spectra were searched against the human International Protein Index (IPI) database (IPI human v3.35 fasta with 62322 entries) using the Bioworks software (Version 3.3.1; Thermo Electron Corp.), based on the Sequest algorithm. The search parameters included (i) precursor ion mass tolerance less than $0.005 \%$; (ii) fragment ion mass tolerance less than $1 \mathrm{Da}$; (iii) up to three missed tryptic cleavages allowed; and (iv) amino acid modifications cysteine carboxyamidomethylation (plus 57.05 Da), and methionine oxidation (plus $15.99 \mathrm{Da}$ ). The corresponding reversed sequence database was used to generate score criteria that yielded an estimated FP (False Positive) rate of 5\% (precision of 0.95). To minimize false positives, all output results were combined together using in-house software to generate score criteria: The cross-correlation scores (Xcorr) of matches were greater than 2.81 and 3.39 for charged state 2 and 3 peptide ions, respectively. To obtain reliable protein identification, only peptides with a $\Delta \mathrm{Cn}$ score above 0.1 , and whose ranks of the primary scores (Rsp) were less than 4 , were used [20,21]. In addition, proteins were identified from two or more distinct peptides, which could be considered as a high-confidence identification.

\subsection{Bioinformatics analysis}

The theoretical isoelectric point (pI), molecular weight (MW) and GRAVY (grand average of hydropathy) values were calculated using software developed in-house. The secretion pathways of the identified proteins were predicted by SecretomeP [22] (free online at http://www.cbs.dtu.dk/ services/SecretomeP). The mapping of putative transmembrane domains in identified proteins was carried out using the transmembrane hidden Markov model (TMHMM) algorithm, available at http://www.cbs.dtu.dk/services/TMHMM [23].

\section{Results and discussion}

\subsection{Verification of secreted proteins by Western blot- ting}

To reduce contaminants from cytoplasmic proteins and harvest pure secreted proteins, all the procedures descried in Cell culture were carried out stringently. In addition, we examined the distribution of $\beta$-tubulin, an abundant cytoskeletal protein in the two fractions. As shown in Figure 1, $\beta$-tubulin was clearly detected in the total cell extracts, but not in the CM. These observations imply that proteins recovered from the culture supernatants were not the result of cell death.

\subsection{Comparison of extraction methods}

In terms of handling, dialysis was the simplest, but the most time-consuming $(2 \mathrm{~d})$. Ultrafiltration was the second most time-consuming method: It suffered from blockage of the column during centrifugation and the loss of low molecular weight protein. However, using precipitation concentration, the resulting precipitates were difficult to dissolve for further analysis, even if a high concentration of the urea solution was used. In terms of efficiency of extraction, the protein yields of these three methods were different. From $20 \mathrm{~mL}$ of culture supernatants, 70, 92.5, and $32.5 \mu \mathrm{g}$ proteins were extracted by precipitation, centrifugal ultrafiltration, and dialysis, respectively. Thus, centrifugal ultrafiltration appeared to be the best in terms of extraction efficiency.

As shown in Figure 2, 360 unique proteins were identified in total. 206, 290, and 157 proteins were yielded by ultrafiltration, precipitation, and dialysis, respectively. Among them, 106 proteins were identified by all three

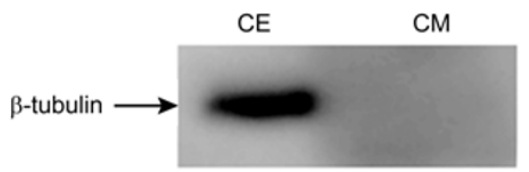

Figure $1 \beta$-tubulin identification by Western blotting with the anti- $\beta$-tubulin antibody. Proteins $(30 \mu \mathrm{g})$ from cell extracts (CE) and conditioned medium $(\mathrm{CM})$ were analyzed.

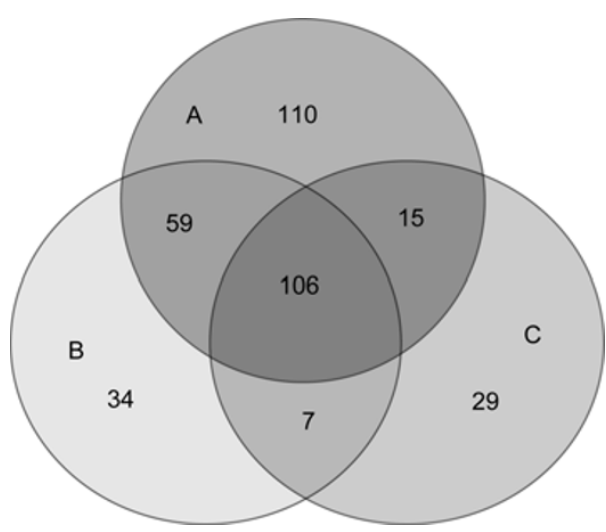

Figure 2 A total of 360 unique proteins were identified by different concentration methods. Ultrafiltration, precipitation, and dialysis yielded 206, 290, and 157 proteins, respectively, with 106 proteins being identified by all three methods. 34,110 , and 29 proteins were uniquely identified in extracts prepared by ultrafiltration, precipitation, and dialysis, respectively. 
methods. Ultrafiltration, precipitation, and dialysis yielded 110, 29, and 34 proteins, respectively, which were unique to that method. Thus, based on the number of proteins identified, precipitation had an advantage and these three concentration methods could supplement each other. The concentration method that produced each identified protein is indicated in Appendix Table S1 in the electronic version.

To assess possible analytical bias of each method, we calculated the MW, pI, and GRAVY values of each protein, based on its primary amino acid sequence. The 290 proteins harvested by precipitation showed a typical molecular weight distribution $(8-630 \mathrm{kD})$, with a maximum of $28 \%$ below $30 \mathrm{kD}$ (Figure 3A); the smallest MW was $8 \mathrm{kD}$. By contrast, $19 \%$ of proteins from the dialysis method were below $30 \mathrm{kD}$. Thus, for the enrichment of low molecular weight proteins, the precipitation method is superior to the other two methods. Figure 3B shows the distribution of the pI values for the three methods: The proteins cover a wide pI range and even very basic proteins (up to a pI of 11.86) can be identified. The distribution of the $\mathrm{pI}$ values for the 360 identified proteins is consistent with previous descriptions of the Golgi compartment [24]. Figure 3C shows that the secretome from precipitation presents the most extensive distribution of GRAVY values and includes more hydrophilic proteins. The overall mean of the GRAVY values was -0.386 between the cytoplasm and endoplasmic reticulum, the values of which were -0.50 and -0.075 , respectively, in a previous study. Of all proteins studied, $5 \%$ had positive GRAVY values indicating a hydrophobic nature.

\subsection{Data analysis}

\subsubsection{Secretory pathway categories of identified proteins}

The identified proteins were further analyzed using bioinformatics software to predict protein secretion pathways. Seventy-eight proteins were predicted by SecretomeP to be released through the nonclassical secretory pathway. Ninety-seven proteins were predicted to be secreted by the classical secretory pathway, which is characterized by the presence of a signal peptide and absence of transmembrane domains [25,26]. In addition, 36 integral membrane proteins were predicted by TMHMM that could not be categorized in the classical or nonclassical secretion pathways. Collectively, these analyses predicted that at least $58.6 \%$ (211/360) of the identified proteins could be released into the $\mathrm{CM}$ of cultured cancer cells via different mechanisms. These 360 classified proteins are all displayed in Appendix Table S1 in the electronic version.

\subsubsection{Identification of known or novel secretory proteins}

Altogether, from the three methods, 360 proteins were confidently identified. Although 360 is not a large number, the efficiency of identification in our study was better than that in a previous study [9], which analyzed a human hepatoma cell, HepG2, using two-dimensional liquid chromatography
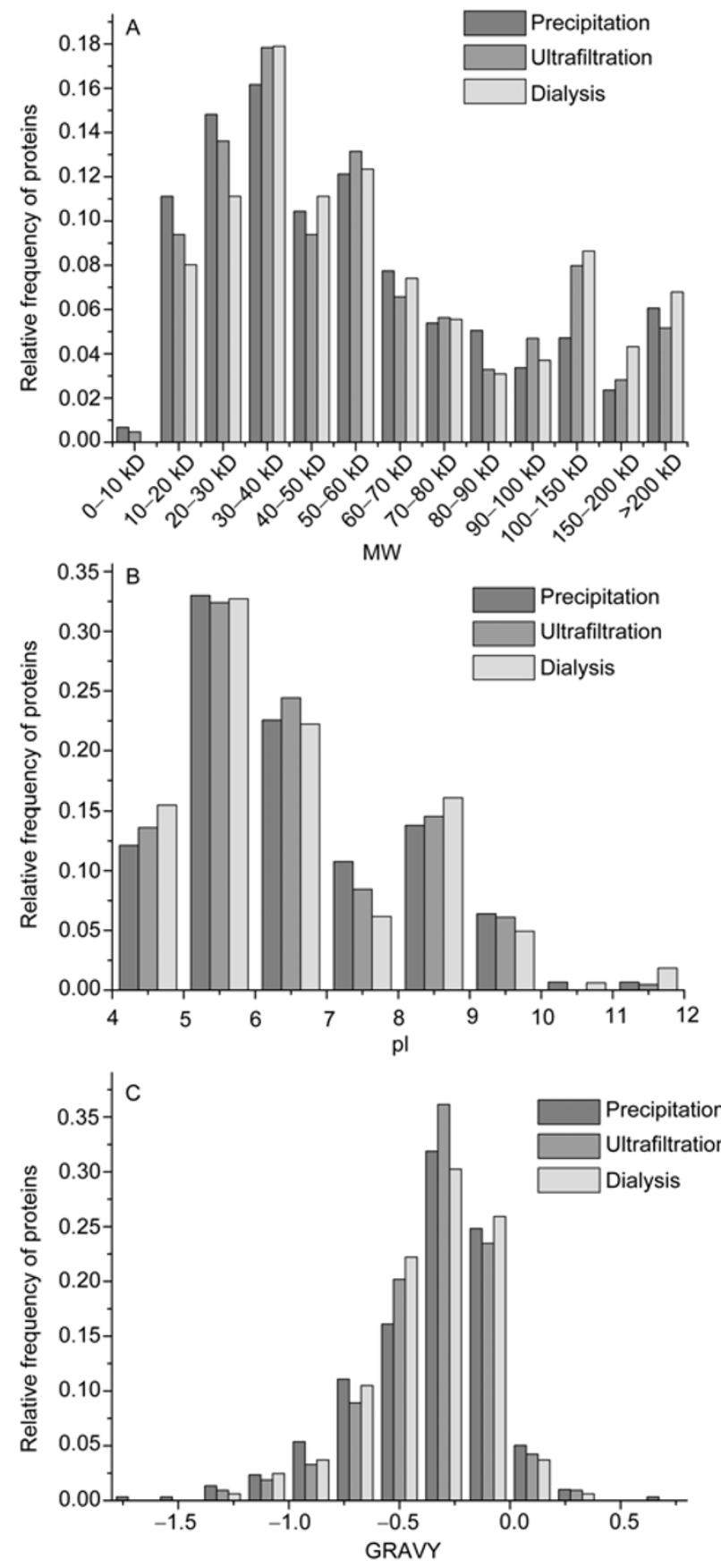

Figure 3 Comparisons of protein distributions based on their physiochemical characteristics. A, MW; B, pI; C, GRAVY among the proteins identified from the three methods.

coupled with tandem mass spectrometry. In that study, only 86 proteins were identified. In addition, in our previous work [27,28], 187 secretory proteins were identified using a glycoproteomics strategy, and 872 secretory proteins were identified using a novel nanozeolite-driven enrichment approach, followed by SDS-PAGE and LC-MS/MS. In the present work, 211 secretory proteins were identified, of which 42 were newly identified, compared with our two previous studies. Furthermore, compared with the dataset 
Rank0 of SPD, a web-based secreted protein database [29], this work led to identification of some known, as well as novel, secreted proteins. Among the 42 newly identified secretory proteins, 11 proteins were known secreted proteins in UniProtKB/Swiss-Prot; however, the other 31 proteins were not annotated as secreted proteins in that database. Thus, the 31 secretory proteins were newly identified in this study, and are all marked with symbols in Appendix Table S1 in the electronic version.

\section{Conclusion}

Comprehensive recovery of secreted proteins is a very challenging task using available extraction strategies. All three strategies applied in this study are widely used. No previous study has compared all three methods to determine which is the best. There was one previous study [18] by Chevallet et al., which compared ultrafiltration and precipitation, but not the dialysis method. They concluded that the lauroyl sarcosinate-TCA precipitation method was better, and did not mention any advantages of ultrafiltration. Our study is the first to compare all three extraction methods, without bias. We found that (i) ultrafiltration generated a higher protein yield than precipitation; (ii) for the enrichment of low molecular weight proteins and quite hydrophilic proteins, precipitation is superior to the other two methods; and (iii) using the number of proteins identified, we concluded that precipitation has the advantage, but these three concentration methods could complement each other.

This work was supported by the National Natural Science Foundation of China (Grant No. 209750240), and the National Basic Research Program of China (Grant No. 2010CB912700).

1 Welsh J B, Sapinoso L M, Kern S G, et al. Large-scale delineation of secreted protein biomarkers overexpressed in cancer tissue and serum. Proc Natl Acad Sci USA, 2003, 100: 3410-3415

2 Huang C M, Ananthaswamy H N, Barnes S, et al. Mass spectrometric proteomics profiles of in vivo tumor secretomes: Capillary ultrafiltration sampling of regressive tumor masses. Proteomics, 2006, 6: 6107-6116

3 Mbeunkui F, Metge B J, Shevde L A, et al. Identification of differentially secreted biomarkers using LC-MS/MS in isogenic cell lines representing a progression of breast cancer. J Proteome Res, 2007, 6: 2993-3002

4 Ye Q H, Qin L X, Forgues M, et al. Predicting hepatitis B virus-positive metastatic hepatocellular carcinomas using gene expression profiling and supervised machine learning. Nat Med, 2003, 9: 416-423

5 Gupta G P, Massagué J. Cancer metastasis: Building a framework. Cell, 2006, 127: 679-695

6 Tang Z Y, Ye S L, Liu Y K, et al. A decade's studies on metastasis of hepatocellular carcinoma. J Cancer Res Clin Oncol, 2004, 130: 187-196

7 Diehl H C, Stuhler K, Volmer M W, et al. A catalogue of proteins released by colorectal cancer cells in vitro as an alternative source for biomarker discovery. Proteomics Clin Appl, 2007, 1: 47-61
8 Pellitteri-Hahn M C, Warren M C, Didier D N, et al. Improved mass spectrometric proteomic profiling of the secretome of rat vascular endothelial cells. J Proteome Res, 2006, 5: 2861-2864

9 Yamashita R, Fujiwara Y, Ikari K, et al. Extracellular proteome of human hepatoma cell, HepG2 analyzed using two-dimensional liquid chromatography coupled with tandem mass spectrometry. Mol Cell Biochem, 2007, 298: 83-92

10 Zwickl H, Traxler E, Staettner S, et al. A novel technique to specifically analyze the secretome of cells and tissues. Electrophoresis, 2005, 26: 2779-2785

11 Trost M, Wehmhöner D D, Kärst U, et al. Comparative proteome analysis of secretory proteins from pathogenic and nonpathogenic Listeria species. Proteomics, 2005, 5: 1544-1557

12 Chan X C, McDermott J C, Siu K W. Identification of secreted proteins during skeletal muscle development. J Proteome Res, 2007, 6: 698-710

13 Sardana G, Marshall J, Diamandis E P. Discovery of candidate tumor markers for prostate cancer via proteomic analysis of cell culture-conditioned medium. Clin Chem, 2007, 53: 429-437

14 Lou X M, Xiao T, Zhao K, et al. Cathepsin D is secreted from M-BE cells: Its potential role as a biomarker of lung cancer. J Proteome Res, 2007, 6: 1083-1092

15 Wu C C, Cheng H C, Chen S J, et al. Identification of collapsin response mediator protein-2 as a potential marker of colorectal carcinoma by comparative analysis of cancer cell secretomes. Proteomics, 2008, 8: 316-332

16 Li Y, Tang Z Y, Ye S L, et al. Establishment of a hepatocellular carcinoma cell line with unique metastatic characteristics through in vivo selection and screening for metastasis-related genes through cDNA microarray. J Cancer Res Clin Oncol, 2003, 129: 43-51

17 Mbeunkui F, Fodstad O, Pannell L K. Secretory protein enrichment and analysis: An optimized approach applied on cancer cell lines using 2D LC-MS/MS. J Proteome Res, 2006, 5: 899-906

18 Chevallet M, Diemer H, Dorssealer A V, et al. Toward a better analysis of secreted proteins: The example of the myeloid cells secretome. Proteomics, 2007, 7: 1757-1770

19 Pan T T, Fang C, Gang Y Z, et al. Subproteomic analysis of the cellular proteins associated with the $3^{\prime}$ untranslated region of the hepatitis $\mathrm{C}$ virus genome in human liver cells. Biochem Biophys Res Commun, 2006, 347: 683

20 Callister S J, Dominguez M A, Nicora C D, et al. Application of the accurate mass and time tag approach to the proteome analysis of sub-cellular fractions obtained from rhodobacter sphaeroides 2.4.1. aerobic and photosynthetic cell cultures. J Proteome Res, 2006, 5: 1940-1947

21 Chen M, Ying W T, Song Y P, et al. Analysis of human liver proteome using replicate shotgun strategy. Proteomics, 2007, 7: 2479

22 Bendtsen J D, Jensen L J, Blom N, et al. Feature-based prediction of non-classical and leaderless protein secretion. Protein Eng Des Sel, 2004, 17: 349-356

23 Krogh A, Larsson B, Heijne G, et al. Predicting transmembrane protein topology with a hidden markov model: Application to complete genomes. J Mol Biol, 2001, 305: 567-580

24 Ho E, Hayen A, Wilkins M R. Characterisation of organellar proteomes: A guide to subcellular proteomic fractionation and analysis. Proteomics, 2006, 6: 5746

25 Antelmann H, Tjalsma H, Voigt B, et al. A proteomic view on genomebased signal peptide predictions. Genome Res, 2001, 11: 1484-1502

26 Zhang Z, Henzel W J. Signal peptide prediction based on analysis of experimentally. Protein Sci, 2004, 13: 2819-2824

27 Cao J, Shen C, Wang H, et al. Identification of N-Glycosylation sites on secreted proteins of human hepatocellular carcinoma cells with a complementary proteomics approach. J Proteome Res, 2009, 8: 662-672

28 Cao J, Hu Y, Shen C, et al. Zeolite LTL nanocrystal-driving high efficient enrichment of secretory proteins in human hepatocellular carcinoma cells. Proteomics, 2009, 9: 4881-4888

29 Chen Y J, Zhang Y, Yin Y, et al. SPD: A web-based secreted protein database. Nucleic Acids Res, 2005, 33: 169-173

Open Access This article is distributed under the terms of the Creative Commons Attribution License which permits any use, distribution, and reproduction in any medium, provided the original author(s) and source are credited. 